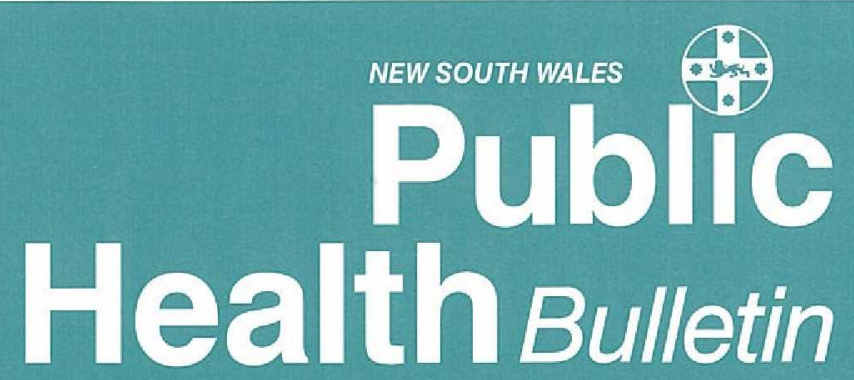

\section{THE NSW HEALTH OUTCOMES INITIATIVE}

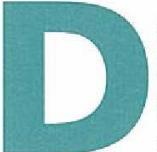

iminishing resources and rapidly expanding and expensive health technologies are putting health systems under increasing pressure to improve cost-effectiveness in the delivery of health services. At the same time the general community continues to be confronted by a confusing array of healthrelated messages. Consumers are asked to make choices about their health care for which there may be little information about those interventions which will yield the best results. Governments remain unconvinced that health care is consistently good value and are cognisant of wide variations in practice styles between geographic areas, without corresponding differences in health outcomes. Providers are concerned that the fiscal imperatives underpinning government policy and decisionmaking will jeopardise the care of their patients.

In response to this climate and under the banner of the NSW Health Outcomes Initiative, the Health Department has turned its attention toward improving the health system's knowledge of the relative outcomes and costs of health services ranging from diagnosis and treatment to prevention. Within the framework of appropriate care, the Health Outcomes Initiative is designed to maximise the population's health and health service outcomes through prevention and clinical services provided within existing resources and in a way that is valued by those receiving care. Appropriate care is defined as that set of health services which maximises the potential health benefits and quality of life of individuals and the population after considering the likely outcomes and cost of each component of the service set. Health care professionals must be willing to provide the set of services and it must be valued by the community.

The framework provides a model which takes account of the efficacy, effectiveness and efficiency of health interventions, empowers providers and patients to make decisions, and acknowledges that the health system is in a constant state of change or evolution. It also provides a means by which the equitable distribution of health services may be possible.

To facilitate development of the framework, it is proposed to establish a process whereby outcome goals and targets, progress indicators and appropriate practice guidelines are established for specified health streams and substreams ${ }^{2}$.

This process will involve the establishment of a steering committee to oversight the implementation and coordination of the Health Outcomes Initiative, including the forming of small, limited-duration panels in the key health stream areas. The steering committee will comprise departmental, professional and consumer representatives. The working parties will comprise representatives of the Health Department and professional colleges as well as expert clinicians, a health economist, a consumer representative and other government departments (where appropriate). The panels will be responsible for the production of goals

\section{Contents}

\section{Articles}

25

The NSW Health

Outcomes Initiative

27 Neonatal Hepatitis $B$ vaccination program

29 Jarman 8: an index of social disadvantage

Infectious diseases

30 Cases of malaria notified in NSW in 1990

32 Cryptosporidium: a summer-autumn epidemic?

33 HIV in NSW changing patterns in risk factors

\section{Notifications}

\section{Correspondence}

Please address all correspondence and potential contributions to:

The Editor,

NSW Public Health Bulletin, Public Health Division, NSW Health Department Locked Bag No 961, North Sydney NSW 2059 Telephone: (02) 3919219

Facsimile: (02) 3919232 


\section{The NSW Health Outcomes Initiative}

\section{Continued from page 25}

and targets for the priority health streams and for the development of appropriate practice guidelines, goals and targets and progress indicators for key substreams. Both the steering committee and the working parties will be supported by a small secretariat and have recourse to a Health Economics Advisory Group for advice on measuring the marginal costs and benefits of health interventions under consideration.

This framework acknowledges that health service managers and providers are primarily concerned with the outcomes of the services for which they are responsible and that the achievement of health outcomes at the population level and many risk factors remain outside the direct control of the health system. The framework facilitates the development of outcome measures which are relevant at the service level (for example, condition-specific complication rates for particular interventions) and at the population level (Area/Region-specific and subgroup morbidity and mortality rates) and in the short and long term. Implementation of this framework will improve the accountability of providers and managers for the services for which they are responsible and the degree to which the quality of health care and overall outcomes of health and nonhealth services are monitored.

The Health Outcomes Initiative should be able to deliver many benefits to both service providers and their clients, including:

\section{- improvements in health outcomes and} increases in the level of appropriate services available to the population within existing resources;

improvements in the information available to make decisions concerning the appropriate allocation of resources and balance of health services; and improvements in monitoring the use and quality of health services at the State and local level through quality assurance mechanisms;

more informed choices about the comparative effectiveness and costs of health interventions by providers, managers and the community; consequent reductions in the use of unnecessary and less effective interventions resulting from the application of guidelines for hospital admissions and appropriate practice.

To stimulate discussion on the Health Outcomes process, the Health Department has identified ten possible health streams (listed below) which it believes worthy of initial consideration, with a further subset of five (with asterisks) which it believes should receive priority consideration:
- serious injury*

- chronic respiratory disease*

- adverse pregnancy outcomes and child development*

- infectious diseases*

- mental disorders*

- cardiovascular disease

- cancers

- poisoning

- diabetes

- chronic musculoskeletal conditions

These health streams essentially fall into the ICD9 rubrics and have been selected because they meet one or more of the following criteria:

- a common cause of suffering, premature death, disability or community concern;

- a major cost to the community, both directly and indirectly (e.g. road trauma through lost years of productive life); and

- an effective prevention or treatment method is available to prevent or control it, and refocusing resources is likely to achieve much.

As the former editor of the New England Journal of Medicine, Arnold Relman, recently stated: "[w]e can no longer afford to provide health care without knowing more about its successes and failures. The Era of Assessment and Accountability is dawning at last; it is the third ${ }^{3}$ and latest - but probably not the last - phase of our efforts to achieve an equitable health care system, of satisfactory quality, at a price we can afford"4.

Over the next few months, representatives of the Department will be consulting with health professional colleges and associations, consumer and community groups and other government departments about the proposed framework. The Health Outcomes Initiative is a long-term project whose success will require the commitment and cooperation of all those involved in the health system.

Further details on the Health Outcomes Initiative may be obtained from Alix Goodwin, Epidemiology and Health Services Evaluation Branch. Phone (02) 3919216.

\section{Alix Goodwin}

Manager, Health Outcomes Initiative

Epidemiology and Health Services Evaluation Branch

1. Harvey R. Making it Better. Strategies for improving the effectiveness and quality of health services in Australia. National Health Strategy Background Paper No.8. 1991; 12.

. A health stream is a set of strategies, health interventions and resources aimed at improving health status in a particular area which is a common cause of suffering, premature death, disability or community concern such as cardiovascular disease and mental health.

3. Relman AS. Assessment and Accountability: The Third Revolution in Medical Care. New Eng J Med 1988; 1220-1222. The first revolution described by Relman was the Era of Expansion which began in the late $1940 \mathrm{~s}$ and early $1950 \mathrm{~s}$ and continued through the $1960 \mathrm{~s}$. The second revolution was the Era of Cost Containment.

4. Op. cit. 